\title{
COMPARISON OF CONDOR, FCI AND MAFIA CALCULATIONS FOR A 150MW S-BAND KLYSTRON WITH MEASUREMENTS
}

\author{
U.Becker, T.Weiland, TH-Darmstadt, Schlossgartenstr. 8, 64289 Darmstadt, Germany \\ M.Dohlus, DESY, Notkestr. 85, 22603 Hamburg, Germany \\ S.Lütgert, Philips, Stresemannallee 101, 22529 Hamburg, Germany \\ D.Sprehn, SLAC, Stanford, CA 94309, USA
}

\begin{abstract}
To facilitate the design of high power klystrons an investigation into the reliability and accuracy of three modern particle-in-cell codes was performed. A 150MW S-band klystron for which measurements were available was used for this comparison. The field calculations of the particle-in-cell codes are based on a finite difference time domain scheme, and use a port approximation to speed up the convergence to steady state. However they differ in many details (eg. calculation of $\mathbf{E}, \mathbf{B}$ or $\mathbf{A}, \phi$; space charge correction; 2D or 3D modelling of output cavity).
\end{abstract}

\section{INTRODUCTION}

The developement of high power klystrons requires computer programs simulating the complex interaction of electrons with electromagnetic fields as realistic as possible. To analyse and verify the abilities of three modern codes (CONDOR, FCI and MAFIA) their calculations are compared with measurements at the 150-MW S-band klystron, which has been designed and built at SLAC [1] for the SBLC test facility at DESY. The design of the 7 cavity klystron is based on CONDOR calculations.

\begin{tabular}{|l||c|c|}
\hline & $\begin{array}{c}\text { design } \\
\text { values }\end{array}$ & $\begin{array}{c}\text { operation } \\
\text { values }\end{array}$ \\
\hline \hline Beam voltage & $535 \mathrm{kV}$ & $527 \mathrm{kV}$ \\
\hline Beam current & $700 \mathrm{~A}$ & $680 \mathrm{~A}$ \\
\hline RF Pulsewidth, rep rate & $3 \mu \mathrm{s}, 60 \mathrm{~Hz}$ & $3 \mu \mathrm{s}, 60 \mathrm{~Hz}$ \\
\hline RF output power & $150 \mathrm{MW}$ & $>150 \mathrm{MW}$ \\
\hline Saturated gain & $55 \mathrm{~dB}$ & $54 \mathrm{~dB}$ \\
\hline Efficiency & $\geq 40 \%$ & $42 \%$ \\
\hline Center frequency & $2998 \mathrm{MHz}$ & $3002 \mathrm{MHz}$ \\
\hline Solenoidal focusing field & $0.21 \mathrm{~T}$ & $0.18 \mathrm{~T}$ \\
\hline
\end{tabular}

\section{COMPUTER CODES}

State of the art are two dimensional particle-in-cell (PIC) codes as CONDOR, FCI and MAFIA-TS2. These codes use the fact that with exception of input and output cavities most klystrons have a pure symmetry of revolution. In these codes the beam is simulated by macroscopic ring charges (macro particles) and their motion is integrated numerically (in 5D phase space). Therefore the electromagnetic fields or their potential representations are calculated in time-domain and take into account: electrostatic fields (eg. gun or dc beam), magnetostatic fields (eg. solenoid), resonant fields (cavities), transient processes and self-consistent field particle interactions. Problems are: the long simulation time (many periods until steady state), the noise caused by macro particles (gain $>55 \mathrm{~dB}$ ), unphysical properties of numerical field calculation and the space charge conservation. The first problem is solved by Yu's port approximation (PAp) [2]: only the beam pipe is simulated, the cavities are represented by their boundary fields, the fundamental mode of each cavity is simulated by an equivalent circuit while the higher modes are neglected. The amplitude of the circuit resonator is controled by a predictor corrector technique to reach steady state in few rf periods. The effect of noise rises with simulation time and is therefore reduced by the fast turning on process. A further reduction is obtained by spatial filtering of fields and current densities and a systematic (not randomized) particle injection. To fulfil the continuity either the mapping of the particle motions to grid currents and the filtering have to be charge conserving (FCI, MAFIA) or the space charge has to be corrected from time to time (CONDOR).

\section{A. CONDOR}

The space charge correction in CONDOR is calculated as a correction potential. Poisson's equation for $\operatorname{div} \vec{D}-\rho$ is solved and the correction is added every nth cycle where $n$ is set by the user. In these calculations $n$ was set between 1 and 3 . The klystron is split into two segments (cavities 1 to 4 and 5,6,7). To transfer particles from one segment to the next, the particle data for the last rf periods are stored in a data dump and when the next segment is started the particles are reinjected periodically. To reduce the noise, the particle data were averaged over four periods. The fields are solved at the split-boundary using Neumann boundaries, no EM fields are dumped, and harmonics travelling in the drift tube are neglected. Therefore, it is best to devide the klystron at a point where the rf currents are relatively small. The output cavity is modelled directly in rz-coordinates (no PAp) whereby the dissipation of the output power is simulated by the $r_{\text {max }}$-boundary.

\section{B. FCI}

For the calculation [3] of the time dependent EM-fields, the wave equation for the scalar and vector potentials $\phi, \mathbf{A}$ in the Lorentz gauge are employed rather than for fields $\mathbf{E}, \mathbf{B}$, because in the potential representation the Lorentz condition is consistent with the continuity equation for charge and current which is automatically satisfied in the simulation. The field distribution of the cavity modes in the drift tube is calculated by the FD method bu using a refined mesh in the cavity region of the drift tube. Particle noise is controlled by a sophisticated filtering algorithm as well as by introducing a small damping term into Maxwell's equations. PAp is used for all cavities. Because the output cavity is of a pill-box type, the field calculation for this cavity has a reduced accuracy. 


\section{MAFIA}

In the 2D simulations the steady state solution for all cavities is derived with the PAp. Therefore, for every dc voltage a special run is necessary to obtain the beam-loading conductances of the cavity modes which are later needed to perform the iteration process of the PAp. Unsymmetric effects and the excitation of higher order modes in the output circuit due to the strong coupling to the external waveguides $\left(\mathrm{Q}_{e x t} \approx 14\right)$ are studied with a 3D PIC-simulation using MAFIA-TS3. The interface plane between the two and three dimensional simulation is located between cavity 6 and 7 . There the periodic particle and electromagnetic field information is stored in the 2D run and reinitialized in the 3D run. The 3D simulation takes into account the cavity with all modes and the real broadband waveguides extracting the power. A detailed description of the interface and other characteristics of klystron simulation using MAFIA is given in [5].

\section{INPUT PARAMETERS OF KLYSTRON CALCULATIONS}

To ensure that the klystron simulations are based on the same operating conditions and to avoid differences caused by precalc. the following parameters have been chosen:

a) Coil Settings: the solenoid field can be adjusted with four independent coils:

\begin{tabular}{|c|c|c|c|}
\hline coil & $\begin{array}{c}\text { current dens. } \\
\mathrm{A} / \text { inch }^{2}\end{array}$ & $\begin{array}{c}\mathrm{Z}_{\min }, \mathrm{Z}_{\max } \\
\text { inch }\end{array}$ & $\begin{array}{c}\mathrm{r}_{\min }, \mathrm{r}_{\max } \\
\text { inch }\end{array}$ \\
\hline \hline bucking & -123.8 & $13.67,17.00$ & $7.97,10.04$ \\
\hline 1 & 1656 & $21.0,73.5$ & $8.34,9.34$ \\
\hline 2 & 1710 & $21.0,73.5$ & $9.84,11.173$ \\
\hline 3 & 1224 & $59.55,72.86$ & $9.84,11.173$ \\
\hline
\end{tabular}

In this $\mathrm{z}$ scale the cathod center is located at $\mathrm{z}=14.415 \mathrm{inch}$. (The field is plotted in [4] and [5].)

b) Beam Voltage and Current: the PIC-simulation starts right behind the gun region, where the DC-current is reinjected according to the particle properties calculated by the codes EGUN (for CONDOR and MAFIA) and SuperSAM (for FCI). The calculated perveances differ less than $2 \%$ from the measured values. For the simulations the current was scaled or extrapolated to the values printed in the $\mathrm{P}_{\text {out }}\left(\mathrm{P}_{\text {in }}\right)$-diagrams.

c) Cold Cavity Parameters: the parameters for the input and output cavity are design values and have been measured. The parameters of the other cavities have been calculated in good agreement by SUPERFISH, SuperLANS and MAFIA-E. The PIC simulations are very sensitive to the cavity parameters especially of cavity 2 and 3 . Therefore the PAp is applied with identical cold cavity parameters in all three PIC-codes. Only the output cavity is simulated directly by MAFIA3D and CONDOR. The frequency and $\mathrm{Q}$ factor of the complete 3D discretization (including coupling slots and waveguides) have been verified in a separate 3D calculation.

\begin{tabular}{|c|c|c|c|c|}
\hline cavity & $\begin{array}{c}\text { frequency } \\
\text { MHz }\end{array}$ & $\begin{array}{c}\mathrm{R} / \mathrm{Q} \\
\text { Ohm }\end{array}$ & $\mathrm{Q}_{l}$ & $\begin{array}{c}\mathrm{z}_{\text {center }} \\
\text { inch }\end{array}$ \\
\hline \hline 1 & 3000 & 87 & 127 & 27.775 \\
\hline 2 & 3009.3 & 89 & 8200 & 33.195 \\
\hline 3 & 3029 & 91 & 8300 & 39.665 \\
\hline 4 & 3176.8 & 107 & 8900 & 55.765 \\
\hline 5 & 3447.8 & 98 & 8700 & 62.695 \\
\hline 6 & 3384.8 & 127 & 9500 & 66.015 \\
\hline 7 & 2998 & 117 & 14 & 70.015 \\
\hline
\end{tabular}

d) Changes: due to a late change of the geometrical description, the FCI calculation are done for a slightly different setup. The geometry of the bucking coil is: $z_{\min }=13$.3inch, $\mathrm{z}_{\max }=16.63$ inch, $\mathrm{r}_{\min }=7$.9inch, $\mathrm{r}_{\max }=9$,975inch. The cathod center is located at 14in, the center of the first cavity at 26inch while the distance between all other cavities is unchanged.

\section{MEASUREMENTS AND COMPARISON \\ A. Measurements}

Only external parameters like input-, reflected-, output-power and the intercepted current could be measured directly. A parasitic oscillation $(\mathrm{f} \approx 8.5 \mathrm{GHz})$ was observed [1] and could be avoided by adjusting the solenoid field (see values in coil settings table). This instability cannot be predicted or analysed by the monopole monomode PAp model.

\section{B. Comparison}

a) Reflected Input Power: essentially the input power is either transfered to the beam or reflected. Neglecting wall losses, one can relate the cavity 1 voltage to the input power, reflection and cold cavity parameters:

$$
V_{1}^{2} / P_{i n}=2(1+r)^{2} Q_{l} R /, Q
$$

which can be solved for $r$ and compared:

\begin{tabular}{|l|c|c|c|c|c|}
\hline $\mathrm{I}_{b} / \mathrm{A}$ & 480 & 495 & 511 & 523 & 527 \\
\hline measur. & & 0.179 & 0.190 & 0.187 & 0.183 \\
\hline CONDOR & 0.169 & 0.216 & 0.179 & & 0.219 \\
\hline FCI & 0.046 & 0.046 & 0.058 & & 0.059 \\
\hline MAFIA & 0.176 & 0.184 & 0.193 & & 0.206 \\
\hline
\end{tabular}

The small reflection of the FCI calculation indicates an inaccurate simulation of the input cavity. The effect of this error is a reduced cavity 1 voltage and therefore a reduced gain $(\approx-1 \mathrm{~dB})$.

b) Linear Gain: the gains calculated by all programs are too high: CONDOR +7.6dB, FCI +4.8dB, MAFIA2D +2.1dB MAFIA3D $+1.8 \mathrm{~dB}$ at operation frequency. In the MAFIA calculation the maximum of the frequency response is shifted by at least $8 \mathrm{MHz}$ to the measurement.

c) Fourier Coefficients of Beam Current: for $\mathrm{U}_{b}=511 \mathrm{kV}$ and identical cavity 1 voltage $(3 \mathrm{kV})$ the fourier components of the beam current and the cavity voltages are documented. In the range of the first three cavities the amplification of the cavity voltages and the 1st harmonic of the current are highest in CONDOR and lowest in MAFIA. The difference between CONDOR and FCI at the beginning is completly compensated to the end of the tube. The amplification along the last drift space is significantly higher in MAFIA so that the cavity 7 voltage is only $2.5 \%$ lower than by the other codes. 
d) Output Cavity $\left(\mathrm{U}_{b}=511 \mathrm{kV}, \mathrm{U}_{1}=3 \mathrm{kV}\right)$ : even with very similar bunching and cavity 7 voltage the output power of CONDOR (155.1MW) and FCI (166.1MW) show a big difference. For these parameters MAFIA2D is in excellent agreement with CONDOR and MAFIA3D is further 10MW lower. The CONDOR/FCI differences and the MAFIA3D/2D differences of $10 \mathrm{MW}$ can only be explained by errors of the PAp. CONDOR (without PAp) and MAFIA2D (with PAp) give similar results because the cav. 7 voltage differs.

e) Numerical Effort:

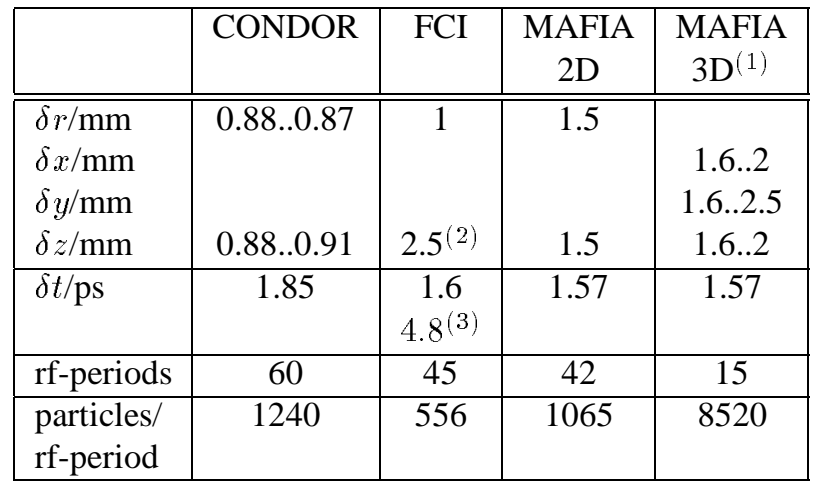

(1) output cavity, (2) increased resolution in the cavity regions,

(3) integration of motion

\section{SUMMARY}

The measured input reflection coefficient indicates that the beam loading of the input cavity is not simulated precisely by FCI. All codes calculate overly high gains (especially CONDOR) and saturated output powers (especially FCI). The linear frequency characteristicas are shifted upwards (especially by MAFIA). The port approximation causes significant errors in the output cavity.

\section{References}

[1] D. Sprehn, R.M. Phillips, G. Caryotakis: "The Design and Performance of 150-MW S-Band Klystrons", SLAC-PUB6677, Stanford Linear Accelerator Center, September 1994.

[2] Simon Yu: "Particle-In-Cell Simulation of High Power Klystrons", SLAC-AP-34, September 1984.

[3] T. Shintake: "High Power Klystron Simulations using FCIField Charge Interaction Code" KEK 90-3 A/D, May 1990.

[4] S. Lütgert: "FCI Parameter Study for the 150 MW DESY Klystron", SBLC meeting at DESY, Hamburg, February 1995.

[5] U. Becker, M. Dohlus, T. Weiland: "Three Dimensional Klystron Simulation", to be published in Particle Accelerators.
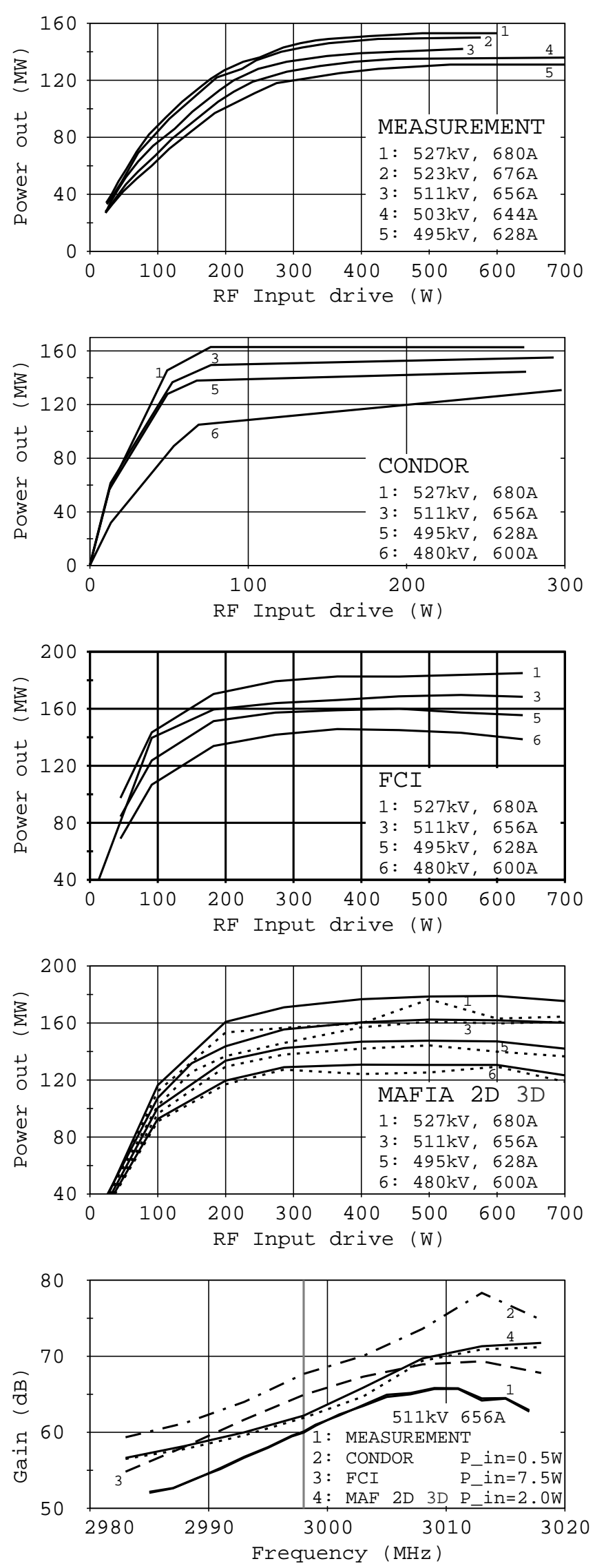